\title{
FRIEDMAN AND SCHWARTZ (1982) REVISITED: ASSESSING ANNUAL AND PHASE-AVERAGE MODELS OF MONEY DEMAND IN THE UNITED KINGDOM
}

\author{
Neil R. Ericsson, David F. Hendry, and Kevin M. Prestwich*
}

March 6, 1998

Abstract: Several studies have developed empirical models of U.K. money demand using the century of annual and phase-average data in Friedman and Schwartz (1982). The current paper evaluates key models from those studies, employing tests of constancy and encompassing. The evidence strongly favors an annual model from Ericsson, Hendry, and Prestwich (1998a), whereas models based on the phase-average data fare poorly.

Keywords: constancy, encompassing, Friedman and Schwartz, money demand.

JEL classifications: E41, C52.

\section{All correspondence should be addressed to the first author (NRE).}

Neil R. Ericsson

Stop 24, Division of International Finance, Federal Reserve Board

2000 C Street, N.W., Washington, D.C. 20551 U.S.A.

(1) (202) 452-3709 (office) (1) (202) 736-5638 (fax)

ericsson@frb.gov (Internet)

David F. Hendry

Nuffield College, Oxford OX1 1NF England

(44) (1865) 278544 (office) (44) (1865) 278557 (fax)

david.hendry@nuffield.oxford.ac.uk (Internet)

Kevin M. Prestwich

AMS - American Management Systems

12601 Fair Lakes Circle, Fairfax, Virginia 22033 U.S.A.

(1) (703) 267-8989 (office) (1) (703) 227-6704 (fax)

kevin_prestwich@mail.amsinc.com (Internet)

${ }^{*}$ The first author is a staff economist in the Division of International Finance, Federal Reserve Board, Washington, D.C., U.S.A. The second author is Leverhulme Personal Research Professor of Economics at Nuffield College, Oxford, England. The third author was a research assistant in the Division of International Finance at the Federal Reserve Board when this paper was initially drafted. The authors may be reached on the Internet at ericsson@frb.gov, david.hendry@nuffield.oxford.ac.uk, and kevin_prestwich@mail.amsinc.com respectively. The views in this paper are solely the responsibility of the authors and should not be interpreted as reflecting the views of the Board of Governors of the Federal Reserve System or of any other person associated with the Federal Reserve System. The first author gratefully acknowledges the generous hospitality of Norges Bank, where he revised some of the material herein. The second author gratefully acknowledges financial support from the U.K. Economic and Social Research Council under grant R000234954. We wish to thank Clifford Attfield, David Demery, and Nigel Duck for compiling the data in Attfield, Demery, and Duck (1995); and Neva Kerbeshian, Helmut Lütkepohl, Jaime Marquez, and two anonymous referees for helpful comments. All numerical results were obtained using PcGive Professional Version 9.00: see Doornik and Hendry (1996). 


\section{Introduction}

Empirical modeling of the demand for money has been a central focus in the economics profession for many years. This interest is reflected in the sequence of studies by Friedman and Schwartz (1982), Hendry and Ericsson (1983), Longbottom and Holly (1985), Escribano (1985), Hendry and Ericsson (1991), Attfield, Demery, and Duck (1995), and Ericsson, Hendry, and Prestwich (1998a), which have developed various empirical models of U.K. money demand using the century of annual and phaseaverage data in Friedman and Schwartz (1982). Those models differ substantially in their empirical properties and in their purported implications for policy, so some model comparison is of value. The current paper evaluates the key models from those studies, employing tests of constancy and encompassing. The evidence strongly favors an annual model from Ericsson, Hendry, and Prestwich (1998a).

These various studies involve different models estimated over different sample periods and on different measures of the data (annual and phase-average). Two types of model comparison are common in the existing literature. First, a given model can be evaluated over different data periods with the same data measure, generating tests of constancy. Second, different models estimated over the same period and with the same data measure can be compared, as with standard encompassing tests. The current paper assesses both constancy and encompassing for three key models that use Friedman and Schwartz's data. It also evaluates these models by a new encompassing approach, comparing different models estimated over the same sample period but with different data measures. Tests of constancy and encompassing are natural to consider for evaluating these models. As Judd and Scadding (1982) document, constancy is a critical and often elusive feature of empirical money-demand equations. Likewise, encompassing is a key feature in a progressive research strategy and so is sensible to consider for extensions of datasets; see Mizon and Richard (1986) and Hendry (1995, Chapters 9 and 14) inter alia.

This paper is organized as follows. Section 2 describes the annual data, and Section 3 discusses the annual models. An annual model in Ericsson, Hendry, and Prestwich (1998a) encompasses previous annual models but not conversely, and that model has empirically constant coefficients. Section 4 defines the relationship between annual and phase-average data and reproduces Friedman and Schwartz's central empirical (phase-average) results on U.K. money demand. Section 5 derives some implications of phase averaging in order to compare the annual and phase-average results. Section 6 then compares annual models from Hendry and Ericsson (1991) and Ericsson, Hendry, and Prestwich (1998a) with the phase-average models from Friedman and Schwartz (1982). The evidence strongly favors the annual models. Throughout, the analysis emphasizes the progressive nature of empirical research and the practical importance of coherent rather than mechanistic extensions of empirical models. 


\section{The Annual Data}

This section describes the annual data. The basic data series are annual values of the broad money stock $(M)$, real net national income $(I)$, the corresponding deflator $(P)$, short-term and long-term nominal interest rates $(R S$ and $R \ell)$, population $(N)$, and high-powered money $(H)$, all for the United Kingdom. Data for 1871 through 1975 are from Friedman and Schwartz (1982). Attfield, Demery, and Duck (1995) extended those series over 1976-1993, constructing them from a variety of sources and splicing together several alternative definitions of money. The variables $M$ and $H$ are in $£$ million; $I$ is in $£$ million for $1929 ; N$ is in millions; $P=1.00$ in 1929 ; and $R S$ and $R \ell$ are fractions. Ericsson, Hendry, and Prestwich (1998b) give further details on the data, and Hendry and Ericsson (1991) and Ericsson, Hendry, and Prestwich (1998a) graph the data and provide some descriptive statistics.

Some constructed variables are of interest. First, under the quantity theory, the income elasticity is unity, so a key derived variable is velocity $V[=(I \cdot P) / M]$.

Second, there are dummy variables. Retaining the notation in Hendry and Ericsson (1991) and Ericsson, Hendry, and Prestwich (1998a), the variables $D_{1}$ and $D_{3}$ are zero-one dummies for World Wars I and II, and $D_{4}$ is a zero-one dummy for 1971-1975. The latter aims to capture the deregulation of the banking sector with the introduction of Competition and Credit Control in 1971. A similar period of deregulation occurs in 1986-1989. As a proxy for both episodes of deregulation, the dummy $D_{c}$ is unity for $1971-1975$ and $1986-1989$, and zero otherwise.

Third, there are several measures for the opportunity cost of holding money $M$. Ideally, the measured opportunity cost would incorporate the own rate of money in addition to the outside rate, but a consistent and complete series on the own rate is not currently available. Three feasible alternatives have been advanced, as follows. Friedman and Schwartz (1982) advocated using a fraction of $R S$, denoted $R N$ and calculated as $(H / M) \cdot R S$. This measure assumes that all components of $M$ except for high-powered money $H$ earn interest at the (outside) short-term rate $R S$. Hendry and Ericsson (1991) propose using the short-term interest rate $R S$ itself because, over Friedman and Schwartz's sample, very little of M2 earned interest, and the interest rate on the interest-bearing component of M2 was itself very low. Finally, Ericsson, Hendry, and Prestwich (1998a) suggested modifying Friedman and Schwartz's measure $R N$ so as to account for the changes in the measurement of highpowered money and broad money that occurred over the extended sample. Proper measurement of $R N$ requires the unspliced data, even while the modeled money series is spliced. To distinguish between measures using spliced and unspliced series, $H$ and $M$ denote spliced series, whereas $H^{a}$ and $M^{a}$ denote actual values (superscript ${ }^{a}$ for actual). Specifically, $H^{a}$ and $M^{a}$ are not rescaled for the definitional changes in 1975 (for $H$ ) and 1987 (for $M$ ). Correspondingly, $R N$ denotes $(H / M) \cdot R S$ (as above) with spliced series, and $R N^{a}$ denotes $\left(H^{a} / M^{a}\right) \cdot R S$. Over $1878-1975, R S$ is virtually 
indistinguishable from $R N$ and $R N^{a}$ (aside from a scale factor) because $H / M$ and $H^{a} / M^{a}$ are essentially constant over that sample period. However, over 1976-1993, $H / M$ and $H^{a} / M^{a}$ plummet from about 0.25 to just under 0.10 (for $H / M$ ) or 0.05 (for $H^{a} / M^{a}$ ), implying very different behavior of $R S, R N$, and $R N^{a}$.

Throughout, capital letters denote both the generic name and the level of a variable; logs are in lowercase; and "levels" often means the logarithm of the levels, with the context clarifying the usage. Uppercase delta $\Delta$ is the difference operator, defined as $(1-L)$, where the lag operator $L$ shifts a variable one period into the past. Hence, for $x_{t}$ (a variable $x$ at time $t$ ), $L x_{t}=x_{t-1}$ and so $\Delta x_{t}=x_{t}-x_{t-1}$. More generally, $\Delta_{j}^{i} x_{t}=\left(1-L^{j}\right)^{i} x_{t}$ for positive integers $i$ and $j$. If $i$ or $j$ is not explicit, it is taken to be unity. Single and double asterisks (* and $\left.{ }^{* *}\right)$ adjacent to values of statistics denote significance at the $5 \%$ and $1 \%$ levels respectively.

\section{Constancy, Encompassing, and the Annual Models}

This section describes models constructed on the initial annual dataset (1871-1975) and then on the extended dataset (1871-1993), paralleling these models' historical development. This progression traces and summarizes the encompassing nature of the model sequence, where encompassing occurs both across samples for a given model (constancy) and across models for a given sample ("standard" encompassing).

Hendry and Ericsson (1983) developed a linear equilibrium correction model (EqCM) over 1878-1970. ${ }^{1}$ Using the same data, Longbottom and Holly (1985) and Escribano (1985) obtained improved specifications, the first through the role of interest rates and the second through a nonlinear EqCM. Each of the two new models encompassed the model in Hendry and Ericsson (1983), but neither could encompass the other. Hendry and Ericsson (1991) developed a model that improved on all three of the previous models, encompassing each one; and they extended the sample through 1975. Because that improved model encompasses the earlier models, it serves like a sufficient statistic for those earlier models. So, for our present purposes, we ignore the earlier models and focus on the improved model, which is an empirically constant EqCM of broad money $M$, in which the quantity theory of money defines the long-run equilibrium and the data determine the short-run dynamics:

$$
\begin{aligned}
& \Delta(m-p)_{t}=\underset{(0.06)}{0.47} \Delta(m-p)_{t-1}-\underset{(0.04)}{0.11} \Delta^{2}(m-p)_{t-2}-\underset{(0.04)}{0.59} \Delta p_{t}
\end{aligned}
$$

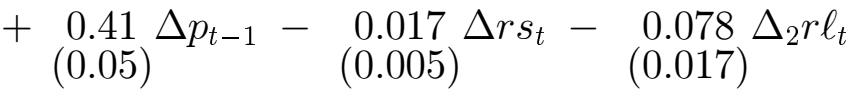

\footnotetext{
${ }^{1}$ Although Hendry and Ericsson (1983), Longbottom and Holly (1985), Escribano (1985), and Hendry and Ericsson (1991) referred to their models as error correction models, technically speaking they are equilibrium correction models. See Hendry (1995, p. 213) for a discussion of the distinction between the two types of models.
} 


$$
\begin{gathered}
-\underset{(0.20)}{1.15}\left(\hat{u}_{t-1}-0.2\right) \hat{u}_{t-1}^{2}+\underset{(0.002)}{0.007}+\underset{(0.6)}{3.4}\left(D_{1}+D_{3}\right)_{t} \\
+\underset{(0.9)}{7.1 D_{4 t}+\underset{(0.027)}{0.090} D_{4 t} \Delta r s_{t}} \\
T=98[1878-1975] \quad R^{2}=0.88 \quad \hat{\sigma}=1.478 \% \text { Jt }=1.22 \quad \text { Var }=0.05 .
\end{gathered}
$$

For each observation, the equilibrium correction residual $\hat{u}$ is calculated as:

$$
\begin{array}{r}
\hat{u}_{t}=(m-p-i)_{t}-\left(-0.310-7.00 R S_{t}\right) \\
T=98[1873-1970] \quad R^{2}=0.56 \quad \hat{\sigma}=10.86 \%
\end{array}
$$

where, for historical reasons, the coefficients in (2) are derived from a static EngleGranger regression over $1873-1970 .^{2}$ Here and below, $t$ is the annual time subscript; $T$ is the number of annual observations; $R^{2}$ is the squared multiple correlation coefficient; $\hat{\sigma}$ is the standard deviation of the residuals, expressed as a percentage of real money and adjusted for degrees of freedom; Jt and Var are Hansen's (1992) statistics for testing joint parameter nonconstancy and variance nonconstancy; and OLS standard errors are in parentheses $(\cdot)$. The coefficients on the war dummies $\left(D_{1}+D_{3}\right)$ and the deregulation dummy $D_{4}$ (and on $D_{c}$, below) have been scaled up 100 -fold so that they are interpretable as percentages. The coefficient on $D_{4 t} \Delta r s_{t}$ is not rescaled, so as to maintain units comparable to those of the coefficient on $\Delta r s_{t}$. While diagnostic tests are important in model evaluation, the papers cited above give a battery of such tests for (1)-(2) and the models examined below, so diagnostic tests (beyond those for constancy and encompassing) are not included herein.

Equations (1)-(2) use the annual data as compiled by Friedman and Schwartz (1982), which end in 1975. Attfield, Demery, and Duck (1995) and Ericsson, Hendry, and Prestwich (1998a, Section III) evaluate mechanistic extensions of this model over 1976-1993 and find strong evidence of parameter nonconstancy. However, the way in which a model is extended over a new sample may bear directly on its statistical performance on that sample. To wit, a coherent economic extension of (1)-(2) over the same sample obtains constant coefficients, as shown in Ericsson, Hendry, and Prestwich (1998a, Section V). This economic extension is:

$$
\begin{aligned}
& \Delta(m-p)_{t}=\underset{(0.05)}{0.48} \Delta(m-p)_{t-1}-\underset{(0.04)}{0.10} \Delta^{2}(m-p)_{t-2}-\underset{(0.04)}{0.62} \Delta p_{t} \\
& +\underset{(0.05)}{0.40 \Delta p_{t-1}}-\underset{(0.006)}{0.020} \Delta r n_{t}^{a}-\underset{(0.016)}{0.041 \Delta} \Delta_{2} r \ell_{t}
\end{aligned}
$$

\footnotetext{
${ }^{2}$ Due to increased numerical accuracy in recent versions of PcGive, the estimated intercept in (2) differs slightly from the one reported in Hendry and Ericsson (1991, equation (9)). See Ericsson, Hendry, and Prestwich (1998a, footnote 4) for details.
} 


$$
\begin{gathered}
-\underset{(0.33)}{2.26}\left(\tilde{u}_{t-1}-0.2\right) \tilde{u}_{t-1}^{2}+\underset{(0.002)}{0.004}+\underset{(0.5)}{3.9}\left(D_{1}+D_{3}\right)_{t} \\
+\underset{(0.7)}{5.2} D_{c t}+\underset{(0.026)}{0.100} D_{4 t} \Delta r s_{t} \\
T=116[1878-1993] R^{2}=0.87 \quad \hat{\sigma}=1.622 \% \quad J t=1.93 \quad \text { Var }=0.82^{* *} \\
\text { Cov }: F(10,95)=1.31 \quad \text { Chow }: F(20,85)=2.73^{* *},
\end{gathered}
$$

where $\tilde{u}$ be the Engle-Granger residual from:

$$
\begin{gathered}
\tilde{u}_{t}=(m-p-i)_{t}-\left(-0.318-6.67 R N_{t}^{a}\right) \\
T=98[1873-1970] \quad R^{2}=0.59 \quad \hat{\sigma}=10.57 \%
\end{gathered}
$$

Equations (1) and (2) require two economic extensions to obtain (3) and (4). First, the deregulation dummy $D_{4}$ is extended as $D_{c}$ when entering by itself in order to capture the deregulation in 1986-1989. Second, the measure of the opportunity cost $(R S)$ is replaced by $R N^{a}$ in order to capture the decline in the non-interest-bearing fraction of money during the last two decades.

The coefficients in (3) and (1) are virtually identical. Forecasts and recursive estimates of (3) in Ericsson, Hendry, and Prestwich (1998a, Figures 7 and 8) and the insignificance of $J t$ and the Chow (1960) covariance statistic (Cov) further confirm the constancy of (3)'s coefficients. That said, $\hat{\sigma}$ has increased by about $15 \%$, and Var and the Chow (1960) predictive-failure statistic (Chow) reject, indicating that the nonconstancy present is due to omitted variables nearly orthogonal to the included variables. Equally, rejection by the predictive-failure and Var tests reflects these tests' high power to detect numerically modest changes in $\hat{\sigma}$. The long sample and the high variance of the data relative to that of the equation error are the proximate reasons for that high power. Overall, the model (3) performs well for this recent turbulent period in the U.K. economy, while its increased error variance reveals that further improvements are possible.

Formal encompassing tests of (1) and (3) strongly favor (3). For the samples considered (1878-1973, 1878-1975, and 1878-1993), the parameter-encompassing statistics are: $F(2,83)=1.22[0.30], F(2,85)=5.28[0.0069]$, and $F(2,103)=22.15[0.0000]$ for whether (1) encompasses $(3)$; and $F(2,83)=0.13[0.88], F(2,85)=0.17$ [0.85], and $F(2,103)=0.45[0.64]$ for whether $(3)$ encompasses $(1) . F(\cdot, \cdot)$ denotes the asymptotic null distribution, and $p$-values are in brackets. This analysis and the more detailed results in Ericsson, Hendry, and Prestwich (1998a) establish that (3) encompasses key aspects of (1) on the extended sample through suitable measurement of the opportunity cost and proper adjustment for financial deregulation. Thus, the following sections turn to assessing these annual models in light of phase-average models. 


\section{Phase-average Data and a Model Thereof}

While the original data are annual, Friedman and Schwartz (1982) analyze phase averages of that annual data in order to focus on the longer-term movements in the data. This section describes what phase averaging is, and it documents Friedman and Schwartz's central phase-average model for U.K. money demand.

In phase averaging, the annual data are averaged separately over contraction and expansion phases of data-selected reference business cycles, where that averaging aims to remove the short-term fluctuations from the data. For a given annual series $\left\{x_{t} ; t=1, \ldots, T\right\}$, the corresponding phase-average series is constructed as:

$$
\bar{x}_{j}=\frac{\frac{x_{t}}{2}+x_{t_{j}+1}+\cdots+x_{t_{j}+c_{j}-1}+\frac{x_{t_{j}+c_{j}}}{2}}{c_{j}} \quad j=1, \ldots, J,
$$

where an overbar denotes phase averaging, $j$ is the index for phase averaging, $c_{j}$ is the phase length of the $j$ th phase (in years), $t_{j}$ is the first year of the $j$ th phase, and $J$ is the number of phase-average observations corresponding to $T$ annual observations. Beginning and ending years are weighted by one-half and appear in adjacent phases as well. Friedman and Schwartz phase-average the logarithms of money, prices, incomes, and population, and the levels of the interest rates.

Friedman and Schwartz (1982, p. 282) present their central regression for U.K. money demand in their Table 6.14. We could closely replicate that equation:

$$
\begin{gathered}
(\bar{m}-\bar{p}-\bar{n})_{j}=\underset{(0.19)}{0.02}+\underset{(0.049)}{0.883}(\bar{\imath}-\bar{n})_{j}-\underset{(3.29)}{11.22} \overline{R N}_{j}-\underset{(0.29)}{0.21} G(\bar{p}+\bar{\imath})_{j} \\
+\underset{(0.58)}{1.38} \bar{W}_{j}+\underset{(2.7)}{20.6} \bar{S}_{j} \\
J=36[1874-1973] \quad R^{2}=0.97 \hat{\sigma}=10.36 \% .
\end{gathered}
$$

The variable $G(\bar{p}+\bar{\imath})$ is the growth rate of nominal income, $\bar{W}$ is a dummy for "postwar adjustment", and $\bar{S}$ is a data-based dummy for "[a]n upward demand shift, produced by economic depression and war ..." during 1921-1955, where quotes are from Friedman and Schwartz (1982, pp. 228, 281). ${ }^{3}$ Friedman and Schwartz also consider a similar equation in rates of change, discussed below.

Hendry and Ericsson (1991) document evidence on the mis-specification of equation (6), including rejection of residual normality, price homogeneity, and constancy, the last by both Chow and covariance statistics. Hansen's statistics provide further

\footnotetext{
${ }^{3}$ The reported numbers in (6) differ slightly from those in Hendry and Ericsson (1991, equation (1)) because of rounding errors in the data. Hendry and Ericsson (1991, equation (1)) uses the phase-average data published in Friedman and Schwartz (1982), which are rounded, whereas (6) uses phase-average data calculated directly from the annual data in Friedman and Schwartz (1982).
} 
evidence of nonconstancy: $J t=2.15^{*}$ and $\operatorname{Var}=0.23$. That said, the performance of (6) relative to (1) and (3) is still of interest, so Sections 5 and 6 interpret and assess the phase-average results in light of their annual counterparts.

\section{An Interpretation of Phase-average Error Variances}

Encompassing statistics are relatively easy to calculate for annual models and for phase-average models. However, encompassing statistics for comparing annual models with phase-average models are as yet undeveloped, although the approach is clear. Using (5), the annual model is reduced to a phase-average representation, whose derived coefficients are compared with the coefficients of the estimated phase-average model. Except under highly restrictive assumptions, the required annual model is a full system of equations for money, prices, income, and interest rates, and not just a conditional money-demand equation. Construction of a full system is beyond the scope of this paper, as is derivation of the formal encompassing statistic.

Section 6 below offers two alternatives: it examines the error variances of annual and phase-average models, and it compares phase-average outcomes with annual outcomes transformed to phase averages. Examination of error variances is of interest because a necessary condition for encompassing is variance dominance, where one equation variance-dominates another equation if the former has a smaller error variance; see Hendry and Richard (1982). To assess variance dominance, the error variances from the two types of models must be in the same units. By re-examining the results in Friedman and Schwartz (1982), the current section shows how to ensure that the variances are in the same units.

In estimating their phase-average regressions, Friedman and Schwartz (1982) used weighted least squares to correct for the heteroscedasticity introduced by variablelength phase-averaging. If unnormalized weights are applied in the phase-average regressions, the resulting equation standard errors are directly comparable to equation standard errors from annual regressions. However, to replicate the regressions in Friedman and Schwartz (1982), the weights must be normalized such that the average weight is unity for regressions in levels, and slightly less than unity for regressions in rates of change. Normalization is immaterial to the validity of the heteroscedasticity transform, but comparisons of the equation standard error for phase-average and annual regressions must account for the normalization factor, which rescales the phase-average equations by a non-unit scalar.

An example clarifies the implications of the normalization. Suppose an annual variable $x_{t}$ is distributed as $I N\left(0, \sigma^{2}\right)$ : that is, $x_{t}$ is serially independent and normal with mean zero and variance $\sigma^{2}$. From (5), the phase average variable $\bar{x}_{j}$ is distributed as $I N\left(0, \sigma^{2} / k_{j}^{2}\right)$, where $k_{j}^{2}=2 c_{j}^{2} /\left(2 c_{j}-1\right)$. Even though the annual variable $x_{t}$ is homoscedastic, the phase average $\bar{x}_{j}$ is not, as its variance depends upon the phase 
length $c_{j}$. To correct this heteroscedasticity, Friedman and Schwartz (1982) used weighted least squares regressions, where the weights depended on $k_{j}$. If the weights had been the $\left\{k_{j}\right\}$ themselves, then the weighted errors in the regression equation would have been distributed as (e.g.) $I N\left(0, \sigma^{2}\right)$ for $\bar{x}_{j}$ regressed on a constant. However, Friedman and Schwartz (1982) [and Attfield, Demery, and Duck (1995) as well] used normalized weights $\kappa_{j}$ (say), where $\kappa_{j}=k_{j} / \bar{k}$ and the normalization factor $\bar{k}$ is the approximate sample mean of $k_{j}$. Because $\bar{k}$ simply rescales the entire weighted least squares regression, the choice between $k_{j}$ and $\kappa_{j}$ does not affect coefficient estimates, their standard errors, or their $t$-ratios. The equation standard error $i s$ affected, though, noting that $\kappa_{j} \bar{x}_{j}$ is distributed as $I N\left(0, \sigma^{2} / \bar{k}^{2}\right)$. Thus, for a phase-average regression estimated by weighted least squares with normalized weights, the equation standard error must be multiplied by $\bar{k}$ to transform it into units comparable to those of the equation standard error from a regression with annual data.

Normalization has consequences for Friedman and Schwartz's phase-average regressions, both in levels and in rates-of-change. For the phase-average equation in levels [comparable to (6)], Friedman and Schwartz (1982, p. 282) report that $\hat{\sigma}$ is $5.54 \%$ from the regression with normalized weights. Because the mean of the unnormalized weights is $\bar{k}=1.825$, the $\hat{\sigma}$ comparable to annual results is $5.54 \% \cdot 1.825=10.11 \%$. That is essentially $10.36 \%$, the value for $\hat{\sigma}$ obtained in (6), using unnormalized weighted least squares. Second, for the phase-average equations with rates of change in (e.g.) Friedman and Schwartz (1982, Table 6.8), the normalization factor $\bar{k}$ is 8.00 exactly, which is close to 7.20 , the average of the unnormalized weights. Thus, for the $\hat{\sigma}$ of $1.34 \%$ in the "final" rates-of-change equation in Friedman and Schwartz (1982, p. 282), the actual $\hat{\sigma}$ comparable to annual results is $1.34 \% \cdot 8.0=10.72 \%$.

\section{Constancy and Encompassing with Different Measures of Data}

Using the adjustments from Section 5, Section 6.1 compares estimated values of $\hat{\sigma}$ from annual and phase-average models for U.K. money demand to determine the direction of variance dominance. ${ }^{4}$ Variance dominance is a property based on in-sample calculations, and variance dominance is necessary for encompassing. Section 6.1 also performs similar comparisons for out-of-sample calculations, where dominance in root mean squared error (RMSE) is necessary for forecast encompassing; see Ericsson (1992). Once comparable units and time periods are obtained, the annual models clearly dominate the phase-average models, both in variance and in RMSE. Then,

\footnotetext{
${ }^{4}$ The situation is further complicated by the data-based selection of the phases, contrasting with the annual model being fit and forecast to data at fixed 1-year intervals. However, Campos, Ericsson, and Hendry (1990) show that the dominant effects of Friedman and Schwartz's phase averaging appear to be captured by fixed-length phase averaging, so the analysis below ignores the consequences of endogenously selecting the phases.
} 
Section 6.2 evaluates the models' statistical and numerical constancy. These combined results allow re-assessment of claims in Friedman and Schwartz (1991) and Attfield, Demery, and Duck (1995).

\subsection{Encompassing and Goodness of Fit}

Table 1 lists comparably scaled values of $\hat{\sigma}$ and RMSE for four equations: the phaseaverage equations in levels and rates of growth from Friedman and Schwartz (1982, p. 282), the initial annual model (1), and the translated annual model (3). This subsection compares these two measures of fit, both across models and across samples.

When estimated over the initial sample of 1878-1973 (equivalent to phases 2$36)$, the annual models (1) and (3) have virtually identical equation standard errors $(1.424 \%$ and $1.406 \%)$ and substantially variance-dominate both phase-average equations, which have equation standard errors of $10.36 \%$ and $10.75 \%$ respectively. The annual models also variance-dominate the phase-average models when estimated over the new sample (1974-1993) and over the extended sample (1878-1993). Almost regardless of sample period or particular model, the phase-average model's equation standard error is 7 or more times that of the corresponding annual model, reflecting just how much better the annual models explain the data relative to the phaseaverage models. Put slightly differently, roughly $98 \%$ of the residual variation in a phase-average model is explained by either annual model.

As this comparison shows, the EqCMs (1) and (3) fit far better than Friedman and Schwartz's phase-average equations in levels and rates-of-change. One potential explanation is the treatment of dynamics: (1) and (3) incorporate dynamics directly, whereas Friedman and Schwartz's equations do not. That said, the latter do implicitly adjust for dynamics through phase averaging, which uses 64 degrees of freedom, contrasting with only 11 degrees of freedom for dynamics in (1) and (3). In that light, comparison of the two phase-average equations with the (static) annual EngleGranger regressions (2) and (4) offers a stark inference. The equation standard errors for those four equations are essentially the same, implying that phase averaging failed entirely in its ostensible purpose - to remove the short-run fluctuations in the data.

The first set of RMSEs in Table 1 are obtained by estimating each model over its initial sample (i.e., phases 2-36, or 1878-1973) and forecasting over the remainder of the sample. For the longest matching forecast sample (phases 37-42, or 19741988), the annual equations dominate the phase-average equations in RMSE. The ratio of RMSEs for (6) and (3) is over 5, similar to the results in-sample. When the forecast sample is extended through 1993, the RMSEs for the annual models increase: marginally for (3) and dramatically for (1). The change in RMSE for (1) arises solely from that equation's poor measure of opportunity cost $(R S)$ and a lack of accounting for financial deregulation. RMSEs for the phase-average models can be computed through only phase 42 (1985-1988) because one regressor in the phase- 
average models is the two-sided growth rate $G(\bar{p}+\bar{\imath})$. Its calculation for phase 43 requires $\bar{p}+\bar{\imath}$ for the (not yet complete) phase 44 (1991 onward). Still, the RMSE over 1974-1993 for the annual model (3) is much smaller than the equation standard errors and RMSEs of the phase-average models over any of the samples.

Choice of units for $\hat{\sigma}$ can affect inference. In Table 1, $\hat{\sigma}$ (and RMSE) for the phaseaverage and annual equations are in comparable units because the underlying phaseaverage regressions used unnormalized weighted least squares. By contrast, Friedman and Schwartz (1982, p. 282) used normalized weighted least squares, and Friedman and Schwartz (1991) did not account for that normalization in their comparisons of phase-average and annual models. Because of this technical error, Friedman and Schwartz (1991, pp. 46-47) incorrectly stated that their regressions variance-dominate those in Hendry and Ericsson (1991). Rather, the converse is true, as Table 1 shows.

The relation between annual and phase-average data provides an additional method for evaluating the annual and phase-average models: transform the annual models' fitted and forecast values to phase averages and compare those values directly with those from the phase-average models. Figure 1 does precisely this for the initial annual model (1) and the phase-average model in levels, (6). Figure $1 a$ plots the actual, fitted, and forecast values over the extended sample (phases 1-43), and Figure $1 c$ plots the corresponding residuals and forecast errors. Figures $1 b$ and $1 d$ are comparable to Figures $1 a$ and $1 c$ but plot values over the forecast sample alone. The phase-average model generally has larger errors than the annual model, an outcome consistent with the comparison of Figures 4 and 7 in Hendry and Ericsson (1991). The phase-average model's forecast error in phase 37 is particularly large, being nearly $20 \%$. Using the transformed annual model (3) rather than the initial annual model (1) obtains an even more pronounced contrast between the annual and phase-average results.

The second set of RMSEs in Table 1 numerically summarize the graphical results in Figure $1 .^{5}$ As with the earlier comparisons, the annual model (1) always dominates the phase-average model (6) for matched samples, reflecting the superior performance of the annual model. Over phases 37-43, the RMSE for the annual model is $13.533 \%$ because of the large outlier in phase 43 (1988-1991). As noted above, the phaseaverage model cannot be tested for that phase.

\subsection{Tests of Constancy}

Tests of constancy are an alternative metric for assessing the relative fit of models, so this subsection analyzes the constancy of the phase-average and annual models. The annual models (1) and (3) both fail the Chow predictive-failure and Hansen Var tests, and (1) fails the covariance test as well: see Table 1 . These failures reflect the models' increased variances over the new and extended samples. By contrast, the

\footnotetext{
${ }^{5}$ Because the annual model used the sample 1878-1970 due to lagged values, its phase-average fitted values begin in phase 2 , not phase 1.
} 
phase-average models show little evidence of nonconstancy over the extended sample.

The apparent contradiction between variance dominance and constancy has an immediate resolution. Variance and RMSE dominance compare the fit of different models across the same sample, whereas the Chow, covariance, and Hansen statistics compare the fit of a given model across different samples. Furthermore, the power of a constancy test depends not only on the magnitude of the change in the coefficients, but also on the fit of the model and on the number of observations available. The annual models fit the data well in sample, their coefficients are precisely estimated, and the forecast period has a large number of observations: 20 for 1974-1993, or about $20 \%$ the number in the initial dataset. Combined, these features ensure that the constancy tests have high power to detect even small nonconstancies in the annual models. Conversely, the phase-average models fit poorly in sample, equation (6) in particular is detectably nonconstant in sample even though that sample is small, and the number of observations forecast is small (only 6). Thus, further nonconstancies in the phase-average model are hard to detect statistically.

Put somewhat differently, the numerical and statistical forecast properties of a model need not be the same: the values of $\hat{\sigma}$, RMSE, and the Chow statistic demonstrate this distinction; see Ericsson (1992). The phase-average model appears constant, in fair part because it fits the data so poorly. The annual model is statistically detectably nonconstant because it fits the data so well.

The distinction between statistical and numerical properties has immediate implications for inferences, as did rescaling $\hat{\sigma}$ in Section 6.1. Specifically, the results in Table 1 and Figure 1 allow a re-assessment of Attfield, Demery, and Duck (1995), which tested for the constancy of (6) over phases 37-42 [1973-1988] and the constancy of (1) over 1976-1993. Using Chow and covariance tests, Attfield, Demery, and Duck (1995, Tables 1-7) found that (6) appears constant whereas (1) does not. In concluding, they claimed:

... the set of estimates based on the phase-average data and the approach adopted by [Friedman and Schwartz (1982)] appear to provide a reasonably good explanation of phase-average money holdings since 1975, at least until the very last phase now available. And some formal tests of stability suggest that these estimates are stable. In contrast, the estimates based on annual observations and the approach adopted by [Hendry and Ericsson (1991)] do not provide a satisfactory explanation for the annual observations after 1975 and appear to be highly unstable. (p. 10)

These inferences are incorrect or misleading, for four reasons. First, while the annual model (1) is statistically less constant than the phase-average model (6), the annual model provides a far better explanation of phase-average money than does the phase-average model; see Figure 1. Attfield, Demery, and Duck (1995) confuse 
goodness of fit and constancy, which are not equivalent concepts. Second, the phaseaverage models appear statistically constant in fair part because they fit the data so poorly. For the phase-average model in levels, tests of constancy out-of-sample are further contaminated because that model is known to be nonconstant in-sample. Third, in Attfield, Demery, and Duck (1995), comparison of annual and phase-average models is based on mismatched samples, biasing the constancy tests in favor of the phase-average models. Their Figures 1a, 1b, and 2 of the models' forecast errors seem impressive, but the worst performance of the annual model is over data that the phase-average model does not attempt to predict. Fourth, Attfield, Demery, and Duck (1995) ignore the economic consequences of redefining the dependent variable. In particular, they mechanically extended the annual model (1) when testing its constancy over 1975-1993. If that model is economically extended, as in (3), it performs quite respectably.

\section{Conclusions}

The central role of money demand relationships in economic policy has stimulated many empirical studies, including those cited above, which developed various empirical models of U.K. money demand using Friedman and Schwartz's annual and phase-average data. This paper evaluates key models from those studies, employing tests of encompassing and constancy. An historical sequence of annual models has led to an equilibrium correction model on data through 1993, where that model encompasses the earlier annual models. That model also captures salient features in the data from 1975 (the end of Friedman and Schwartz's sample) through 1993 - a period with radical changes in economic policy. The model's performance on the new data depends directly upon sensible economic choices for extending the time series of those data to reflect their altered measurement. That said, the annual model, whether mechanistically or economically extended, explains the data far better than the phase-average models. This result holds over the fitted sample and forecast periods; and it holds regardless of whether outcomes are compared across the different datasets on a uniform metric, or whether the outcomes from the annual model are transformed into phase averages and then compared with the outcomes from the phase-average models. The remaining nonconstancy in the economically extended annual model indicates the high power of constancy tests in well-fitting models, and it points to the possibility of further progress in model specification. 
Table 1. Estimated Equation Standard Errors $(\hat{\sigma})$, RMSEs, and Constancy Statistics for Phase-average and Annual Models

\begin{tabular}{|c|c|c|c|c|}
\hline \multirow{2}{*}{$\begin{array}{l}\text { Statistic } \\
\text { [phase observations } \\
\text { in brackets] }\end{array}$} & \multicolumn{2}{|c|}{ Phase-average Data } & \multicolumn{2}{|c|}{ Annual Data } \\
\hline & $\begin{array}{l}\text { Log-level } \\
\text { model (6) }\end{array}$ & $\begin{array}{c}\text { Growth-rate } \\
\text { model }\end{array}$ & $\begin{array}{c}\text { Initial } \\
\text { model (1) }\end{array}$ & $\begin{array}{l}\text { Translated } \\
\text { model (3) }\end{array}$ \\
\hline \multicolumn{5}{|l|}{$\hat{\sigma}$} \\
\hline [2-36] $\quad 1878-1973$ & 10.36 & 10.75 & 1.424 & 1.406 \\
\hline$[37-44] 1974-1993$ & 21.70 & 28.37 & 2.915 & 2.644 \\
\hline$[2-44] \quad 1878-1993$ & 10.95 & 12.67 & 1.931 & 1.622 \\
\hline \multicolumn{5}{|l|}{ RMSE: original data } \\
\hline [37-42] 1974-1988 & 13.93 & 20.76 & 6.750 & 2.649 \\
\hline [37-44] 1974-1993 & - & - & 28.125 & 2.757 \\
\hline \multicolumn{5}{|l|}{ RMSE: Figure 1} \\
\hline$[1-36]$ & 10.15 & - & - & - \\
\hline$[2-36]$ & 10.19 & - & 1.346 & - \\
\hline$[37-42]$ & 14.75 & - & 6.110 & - \\
\hline$[37-43]$ & - & - & 13.533 & - \\
\hline \multicolumn{5}{|l|}{ Chow statistic } \\
\hline value & 1.71 & $3.28^{*}$ & $5.40^{* *}$ & $2.73^{* *}$ \\
\hline$p$-value & 0.1536 & 0.0139 & 0.0000 & 0.0007 \\
\hline degrees of freedom & $(6,30)$ & $(6,29)$ & $(20,87)$ & $(20,85)$ \\
\hline \multicolumn{5}{|l|}{ Covariance statistic } \\
\hline value & 0.30 & 1.03 & $4.95^{* *}$ & 1.31 \\
\hline$p$-value & 0.8736 & 0.4065 & 0.0000 & 0.2342 \\
\hline degrees of freedom & $(4,32)$ & $(4,31)$ & $(10,95)$ & $(10,95)$ \\
\hline \multicolumn{5}{|l|}{ Hansen statistic } \\
\hline$J t$ & 1.71 & 1.10 & 2.58 & 1.93 \\
\hline Var & 0.28 & $0.58^{*}$ & $0.86^{* *}$ & $0.82^{* *}$ \\
\hline
\end{tabular}

Notes:

1. All values of $\hat{\sigma}$ and RMSE are in percent.

2. Listed dates correspond to estimation periods (for $\hat{\sigma}$ ) and forecast periods (for the RMSE).

3. Phase observations appear in square brackets. For $\hat{\sigma}$, the listed phase observations are the literal equivalents to the years listed. The actual samples for the phase-average models are 1-36, 37-42, and 1-42 for the log-level model, and 2-36, 3742 , and 2-42 for the growth-rate model. For the RMSEs, the listed phase observations are the literal equivalents to the years listed and are also the actual samples. RMSEs and $\hat{\sigma}^{\prime}$ s for phase-average samples are converted to annual units for comparability.

4. Statistics for testing constancy are calculated over phases 1-42 and 2-42 for the phase-average equations in levels and growth rates and over 1878-1993 for the annual equations. 

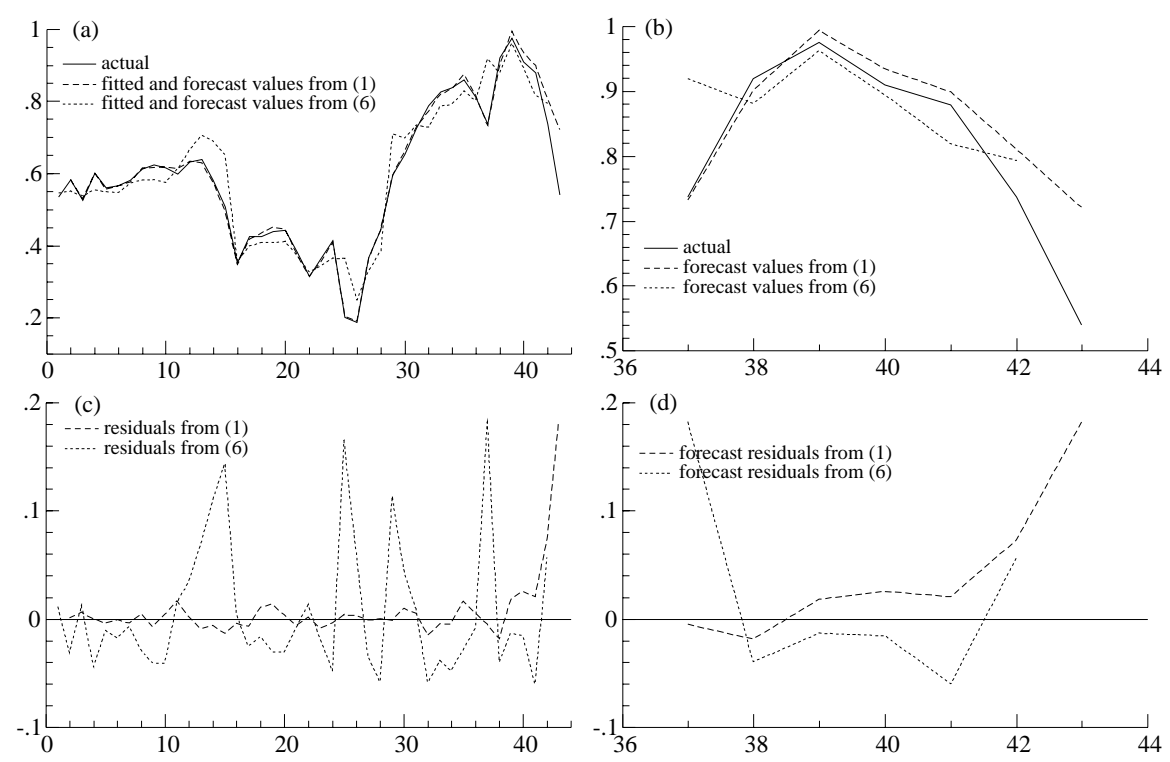

Figure 1: Actual, fitted, and forecast values for velocity, and the corresponding residuals from annual (--) and phase-average (- -) equations in a common phase mapping. 


\section{References}

Attfield, C. L. F., D. Demery, and N. W. Duck (1995) "Estimating the UK Demand for Money Function: A Test of Two Approaches", mimeo, Department of Economics, University of Bristol, Bristol, England, November.

Campos, J., N. R. Ericsson, and D. F. Hendry (1990) "An Analogue Model of Phase-averaging Procedures", Journal of Econometrics, 43, 3, 275-292.

Chow, G. C. (1960) "Tests of Equality Between Sets of Coefficients in Two Linear Regressions", Econometrica, 28, 3, 591-605.

Doornik, J. A., and D. F. Hendry (1996) PcGive Professional 9.0 for Windows, International Thomson Publishing, London.

Ericsson, N. R. (1992) "Parameter Constancy, Mean Square Forecast Errors, and Measuring Forecast Performance: An Exposition, Extensions, and Illustration", Journal of Policy Modeling, 14, 4, 465-495.

Ericsson, N. R., D. F. Hendry, and K. M. Prestwich (1998a) "The Demand for Broad Money in the United Kingdom, 1878-1993", Scandinavian Journal of Economics, 100, 1, forthcoming.

Ericsson, N. R., D. F. Hendry, and K. M. Prestwich (1998b) "Friedman and Schwartz (1982) Revisited: Assessing Annual and Phase-average Models of Money Demand for the United Kingdom", International Finance Discussion Paper No. 61*, Board of Governors of the Federal Reserve System, Washington, D.C., March, available on the WorldWide Web at www.bog.frb.fed.us/pubs/ifdp/1998/61*/default.htm.

Escribano, A. (1985) "Non-linear Error-correction: The Case of Money Demand in the U.K. (18781970)", mimeo, University of California at San Diego, La Jolla, California, December.

Friedman, M., and A. J. Schwartz (1982) Monetary Trends in the United States and the United Kingdom: Their Relation to Income, Prices, and Interest Rates, 1867-1975, University of Chicago Press, Chicago.

Friedman, M., and A. J. Schwartz (1991) "Alternative Approaches to Analyzing Economic Data", American Economic Review, 81, 1, 39-49.

Hansen, B. E. (1992) "Testing for Parameter Instability in Linear Models", Journal of Policy Modeling, 14, 4, 517-533.

Hendry, D. F. (1995) Dynamic Econometrics, Oxford University Press, Oxford.

Hendry, D. F., and N. R. Ericsson (1983) "Assertion Without Empirical Basis: An Econometric Appraisal of 'Monetary Trends in ... the United Kingdom' by Milton Friedman and Anna Schwartz", in Monetary Trends in the United Kingdom, Bank of England Panel of Academic Consultants, Panel Paper No. 22, Bank of England, London, October, 45-101.

Hendry, D. F., and N. R. Ericsson (1991) "An Econometric Analysis of U.K. Money Demand in Monetary Trends in the United States and the United Kingdom by Milton Friedman and Anna J. Schwartz", American Economic Review, 81, 1, 8-38.

Hendry, D. F., and J.-F. Richard (1982) "On the Formulation of Empirical Models in Dynamic Econometrics", Journal of Econometrics, 20, 1, 3-33.

Judd, J. P., and J. L. Scadding (1982) "The Search for a Stable Money Demand Function: A Survey of the Post-1973 Literature", Journal of Economic Literature, 20, 3, 993-1023.

Longbottom, A., and S. Holly (1985) "Econometric Methodology and Monetarism: Professor Friedman and Professor Hendry on the Demand for Money", Discussion Paper No. 131, London Business School, London, England, February.

Mizon, G. E., and J.-F. Richard (1986) "The Encompassing Principle and its Application to Testing Non-nested Hypotheses", Econometrica, 54, 3, 657-678. 